\title{
Conservative multi-implicit spectral deferred correction methods for reacting gas dynamics is
}

\author{
Anita T. Layton *, Michael L. Minion \\ Department of Mathematics, University of North Carolina, Chapel Hill, NC 27599, USA
}

Received 30 January 2003; received in revised form 19 August 2003; accepted 29 September 2003

\begin{abstract}
In most models of reacting gas dynamics, the characteristic time scales of chemical reactions are much shorter than the hydrodynamic and diffusive time scales, rendering the reaction part of the model equations stiff. Moreover, nonlinear forcings may introduce into the solutions sharp gradients or shocks, the robust behavior and correct propagation of which require the use of specialized spatial discretization procedures. This study presents high-order conservative methods for the temporal integration of model equations of reacting flows. By means of a method of lines discretization on the flux difference form of the equations, these methods compute approximations to the cell-averaged or finitevolume solution. The temporal discretization is based on a multi-implicit generalization of spectral deferred correction methods. The advection term is integrated explicitly, and the diffusion and reaction terms are treated implicitly but independently, with the splitting errors reduced via the spectral deferred correction procedure. To reduce computational cost, different time steps may be used to integrate processes with widely-differing time scales. Numerical results show that the conservative nature of the methods allows a robust representation of discontinuities and sharp gradients; the results also demonstrate the expected convergence rates for the methods of orders three, four, and five for smooth problems.
\end{abstract}

(C) 2003 Elsevier Inc. All rights reserved.

Keywords: Conservative methods; Operator splitting; Reactive flows; Semi-implicit methods; Spectral deferred correction methods

\section{Introduction}

Mathematical models that describe the dynamics of reacting flows consist of systems of partial differential equations (PDEs), which specify the advection, diffusion, and reaction of chemical species within a moving medium and which couple the effects of non-reactive hydrodynamics with the effects of heat release

\footnotetext{
This work was supported in part under contract DE-AC03-76SF00098 by the Director, Department of Energy (DOE) Office of Science; Office of Advanced Scientific Computing Research; Office of Mathematics, Information, and Computational Sciences; Applied Mathematics Sciences Program.

${ }^{*}$ Corresponding author. Tel.: +1-919-962-8475; fax: +1-919-962-9345.

E-mail addresses: layton@amath.unc.edu (A.T. Layton), minion@amath.unc.edu (M.L. Minion).
} 
in the chemical reactions. Computational methods intended for solving equations arising in reactive flow models must contend with the fact that the characteristic time scales of the chemical reactions are typically much shorter than the hydrodynamic time scales governed by advection and diffusion. In addition, nonlinear advection or forcing terms may introduce sharp spatial variations into the solutions, the computation of which requires specialized spatial discretization techniques. Thus, the construction of numerical methods that can accurately and efficiently approximate equations with highly disparate time scales and sharp spatial variations in the solution is a difficult and important pursuit.

In this paper, numerical methods are introduced for a particular set of equations that describe reacting compressible flows. If the diffusion and reaction terms in these equations are ignored, they become the standard Euler equations of gas dynamics for which many specialized spatial discretization techniques have been developed. For the Euler equations (as well as other systems of hyperbolic conservation laws), most grid-based numerical methods are cast in conservation form and techniques like Godunov methods [11], essentially non-oscillatory (ENO) methods [25], piecewise parabolic method (PPM) [8], total-variationdiminishing (TVD) methods [23], or wave propagation methods [19] are used to construct conservative flux values, which allow accurate and robust representation of shocks without introducing non-physical oscillations or excessive diffusion into the solution.

When diffusive terms are present, the equations become the compressible Navier-Stokes equations. Diffusive terms can be included into a conservation form of the equations, even though physical quantities are no longer conserved. Generally, diffusive terms regularize the equations so that shocks can no longer form. However, sharp features can still form wherein $\mathrm{O}(1)$ changes in the solution occur within a small spatial region. Therefore the specialized conservative formulations discussed above are still necessary.

The addition of reaction terms to the equations of motion has several ramifications. First, the equations can typically no longer be put in strict conservation form. Moreover, because of the disparity in time scales, the equations for reactive gas dynamics are typically stiff, with the reaction terms much stiffer than the advection and diffusion terms; reaction terms also contribute to the formation of sharp spatial features in the flow, even in cases where the influence of diffusive terms are comparable to that of advective terms.

The most common approach to incorporating reaction terms into numerical methods for compressible gas dynamics is an operator-splitting approach [18,27,28], in which advective and diffusive terms are decoupled from reaction terms. Reaction terms can then be solved using implicit methods without solving fully coupled equations. Moreover, by integrating slower processes using larger time steps, the overall computational cost can be reduced. The drawback of this approach is that temporal accuracy higher than second order is difficult to achieve.

Higher-order temporal accuracy for PDEs is most often achieved through a method of lines (MOL) approach. In this approach, the equations are first discretized in space only, resulting in a system of ordinary differential equations (ODEs). These equations can then be integrated using standard ODE methods. In the present context, because of the stiffness of the reaction and diffusion terms, it is advantageous to handle these terms implicitly so that a prohibitive time step restriction may be avoided. Fully implicit methods [9,12], which treat every term implicitly, require the solution of implicit equations coupling nonlinear advection and reaction; thus, these methods are computationally expensive. In contrast, semiimplicit or implicit-explicit methods can be applied which treat the advection (and possibly diffusion) term explicitly and the reaction (and possibly diffusion) terms implicitly $[2,16]$. When diffusion is significant, treating it explicitly results in a time step restriction, while treating it implicitly and coupling it to the reaction term requires the solution of a nonlinear and non-local equation, which can be computationally expensive, particularly when the number of chemical species is large.

In [4], high-order multi-implicit spectral deferred correction (MISDC) methods are presented for the solution of advection-diffusion-reaction (A-D-R) equations. MISDC methods are a generalization of the spectral deferred correction (SDC) method proposed by Dutt et al. [10]. MISDC methods are similar to operator-splitting methods in that they allow reaction and diffusion terms to be integrated separately, 
implicitly, and with different time steps. However, unlike traditional operator-splitting methods, MISDC methods are easily extended to higher-order temporal accuracy.

In [4], the main focus is on temporal integration; the problems considered all have smooth solutions for which spatial discretization using standard centered differencing is appropriate. In this study, a method is developed which incorporates within the MISDC methods a conservative differencing scheme similar to the PPM developed by Colella and Woodward [8]. The resulting methods - the conservative MISDC methods compute higher-order approximations to the cell-averaged or finite-volume solution of the model equations; the sharp gradients and reaction fronts in the solutions are accurately and robustly represented.

The outline of this paper is as follows. In Section 2, the model equations are described in differential form and in cell-average or finite-volume form. In Section 3, the conservative MISDC methods are first presented in the context of a MOL discretization of the A-D-R equations and then extended to the reacting gas dynamics model. In Section 4, numerical results are presented to demonstrate that the conservative nature of the methods allows a robust representation of discontinuities and sharp gradients, and to demonstrate the expected temporal convergence rates for the methods of orders three, four, and five for smooth problems.

\section{Model equations}

The physical model considered in this study is that of a gaseous mixture in which $M$ chemical species interact in one reaction. The problem is presented in one space dimension; extension to more space dimension can be done by means of finite-volume multi-dimensional approaches [6,20]. As in onedimensional problems, the MOL approach is used to solve multi-dimensional problems: fluxes are computed across cell walls rather than cell edges, and the solution is advanced in time using MISDC methods.

Let $\rho$ be the density, $u$ be the fluid velocity, $E$ be the total specific energy, $p$ be the pressure, $T$ be the temperature, and $Z_{i}$ be the mass fraction of species $i$. With this notation, the compressible Navier-Stokes equations are given by

$$
\begin{aligned}
& \rho_{t}+(\rho u)_{x}=0, \\
& (\rho u)_{t}+\left(\rho u^{2}+p\right)_{x}=\mu u_{x x}, \\
& (\rho E)_{t}+(\rho u E+u p)_{x}=\left(\mu\left(\frac{u^{2}}{2}\right)_{x}\right)_{x}+\frac{R}{\gamma-1}\left(\lambda T_{x}\right)_{x}, \\
& \left(\rho Z_{i}\right)_{t}+\left(\rho u Z_{i}\right)_{x}=\left(D Z_{i} x\right)_{x}+g_{i},
\end{aligned}
$$

where $\mu, \lambda$, and $D$ are diffusion coefficients, $R$ is Boltzmann's gas constant, and $\gamma$ is taken to be 1.4 for air. In this study, reaction terms are assume to take the form

$$
g_{i}=w_{i}\left(v_{p_{i}}-v_{r_{i}}\right) B T^{\alpha} e^{-T_{\mathrm{o}} / T} \prod_{j=1}^{M}\left(\rho Z_{j}\right)^{v_{r_{j}}}
$$

for $i=1, \ldots, M$, where $w_{i}$ is the molecular weight of species $i, B>0$ and $\alpha>0$ are constants, $T_{\mathrm{o}}$ is the ignition temperature for the reaction, and $v_{r_{i}}$ and $v_{p_{i}}$ are the stoichiometric coefficients for species $i$ appearing as a reactant and product, respectively, in the reaction. The model may also be extended to include expressions for more general reaction terms, see [15]. 
The total specific energy $E$ is given by the equation of state

$$
E=\frac{p}{(\gamma-1) \rho}+\frac{u^{2}}{2}+\sum_{j=1}^{M} q_{j} Z_{j},
$$

where $q_{i}$ is the amount of heat released by species $i$. Assuming the perfect gas law, the temperature and pressure are related by

$$
T=\frac{p}{\rho R} .
$$

Traditional finite difference methods, in which spatial derivatives are approximated by means of finite differences, may break down near discontinuities or sharp fronts in solutions. Thus, equations such as (1)(4) are frequently solved using finite-volume methods, which approximate solutions to the cell-averaged or finite-volume form of the equations rather than the differential form.

Before presenting the finite-volume form of (1)-(4), some notation must be defined. In the spatial discretization on the interval $x \in[a, b]$, let $\Delta x$ denote the spatial grid interval. Let $x_{j}$ denote the center of the $j$ th cell $\left[x_{j-\frac{1}{2}}, x_{j+\frac{1}{2}}\right]$, for $j=1, \ldots, N$, where $x_{j-\frac{1}{2}}$ and $x_{j+\frac{1}{2}}$ are the left and right edges of the $j$ th cell, i.e., $x_{j-\frac{1}{2}} \equiv a+(j-1) \Delta x$ and $x_{j+\frac{1}{2}} \equiv a+j \Delta x$. For an arbitrary function $\psi(x)$, let $\bar{\psi}_{j}$ be the cell-averaged value of $\psi(x)$ in the $j$ th cell, i.e.,

$$
\bar{\psi}_{j}=\frac{1}{\Delta x} \int_{x_{j-\frac{1}{2}}}^{x_{j+\frac{1}{2}}} \psi(x) \mathrm{d} x .
$$

Also, let $\psi_{j+\frac{1}{2}}$ denote the numerical approximation to $\psi\left(x_{j+\frac{1}{2}}\right)$.

Consider a differential equation of the form

$$
\psi_{t}=\mathscr{F}_{x}+\mathscr{R},
$$

where $\mathscr{F}_{x}$ corresponds to the advection and diffusion terms, and $\mathscr{R}$ corresponds to the reaction term. The finite-volume form of (9) is given by

$$
\left(\bar{\psi}_{t}\right)_{j}=\frac{\mathscr{F}\left(\psi_{j+\frac{1}{2}}, t\right)-\mathscr{\mathscr { F }}\left(\psi_{j-\frac{1}{2}}, t\right)}{\Delta x}+\overline{\mathscr{R}}_{j} .
$$

Because $\mathscr{R}$ is not in flux form in (9), it is expressed as a cell-averaged variable in (10).

\section{Conservative MISDC methods}

This section first presents a brief description of conservative MISDC methods in the context of A-D-R equations and then extends the methods to the solution of reactive gas dynamics described by (1)-(4). Conservative MISDC methods are similar to the original MISDC methods [4] in that processes of different time-scales are decoupled and integrated using different time steps. However, unlike the original MISDC methods, conservative MISDC methods integrate the advection and diffusion terms in the flux difference form.

\subsection{Advection-diffusion-reaction equations}

Conservative MISDC methods compute approximations to the cell-averaged or finite-volume solution of an A-D-R equation via a MOL discretization on the flux difference form of the equation. Let $u(x, t)$ be a (possibly vector-valued) function that satisfies the A-D-R equation 


$$
u_{t}=\left(f_{\mathscr{A}}\right)_{x}+\mu u_{x x}+\mathscr{R} \equiv \mathscr{A}+\mathscr{D}+\mathscr{R}
$$

for $x \in[a, b]$ and $t \in[0, T]$. In (11), $f_{\mathscr{A}}(t, u(x, t))$ is the advective flux term, $\mu u_{x}$ is the diffusive flux term, and $\mathscr{R}(t, u(x, t))$ is the reaction term. Boundary conditions and initial conditions must be given to complete the specification of the problem. The diffusivity $\mu$ is assumed to be zero or small for the gas dynamics models used in this study. For diffusivity $\mu \neq 0, u$ and $u_{x}$ are continuous for most $f_{\mathscr{A}}$ and $\mathscr{R}$ of interest; however, for a small $\mu$, nonlinear forcings may still introduce sharp gradients that require a specialized spatial discretization approach.

Eq. (11) is rewritten in finite-volume form as described in Section 2. A MOL discretization of the resulting equation yields the following system of ODEs

$$
\begin{aligned}
& \bar{u}_{j}^{\prime}(t)=\overline{\mathscr{A}}_{j}(t, \bar{u}(t))+\overline{\mathscr{D}}_{j}(t, \bar{u}(t))+\overline{\mathscr{R}}_{j}(t, \bar{u}(t)), \\
& \bar{u}_{j}(0)=\left(\bar{u}_{0}\right)_{j},
\end{aligned}
$$

where $\overline{\mathscr{A}}$ and $\overline{\mathscr{D}}$ are given by

$$
\begin{aligned}
& \overline{\mathscr{A}}_{j}(t, \bar{u}(t))=\frac{\left(f_{\mathscr{A}}\right)_{j+\frac{1}{2}}\left(t, u^{*}(t)\right)-\left(f_{\mathscr{A}}\right)_{j-\frac{1}{2}}\left(t, u^{*}(t)\right)}{\Delta x}, \\
& \overline{\mathscr{D}}_{j}(t, \bar{u}(t))=\mu \frac{u_{x_{j+\frac{1}{2}}}(t)-u_{x_{j-\frac{1}{2}}}(t)}{\Delta x} .
\end{aligned}
$$

Because of the MOL approach, $\overline{\mathscr{A}}_{j}(t, \bar{u}(t)), \overline{\mathscr{D}}_{j}(t, \bar{u}(t))$, and $\overline{\mathscr{R}}_{j}(t, \bar{u}(t))$ are evaluated at the integration nodes in time. That is, unlike many second-order conservative methods (e.g., Godunov method [11]), there is no "time-centering" of fluxes or characteristic tracing.

In (14), $u^{*}(t)$ is the solution of the Riemann problem with the left and right states computed from a piecewise parabolic interpolation of $\bar{u}(t)$ [8]. The piecewise parabolic interpolation is fourth-order accurate in regions where the solution is smooth (i.e., where the fourth derivative of the solution exists and is finite). As is done in [8], the interpolation function is set to be a constant if the left or right state is a local maximum or minimum to ensure monotonicity; a narrower profile is used in the interpolation in the neighborhood of a sharp gradient or a discontinuity. The procedures and parameters used to detect sharp gradients or discontinuities are the same as [8]. For $\mu=0, \overline{\mathscr{D}}_{j}=0$; for $\mu \neq 0, u_{x}$ is continuous but higher-order derivatives may be discontinuous. Thus, $u_{x}$ in (15) is approximated using a fourth-order centered difference in regions where the solution is smooth; a second-order centered difference is used in the neighborhood of a sharp gradient.

The reaction term $\mathscr{R}$, which is not in flux form in (11), is expressed as a cell-averaged variable in (12). To achieve high-order spatial accuracy, $\mathscr{R}$, which is nonlinear, is computed as point values at cell edges. The point values $\mathscr{R}_{j \pm \frac{1}{2}}$ are then used to update cell-averages $\overline{\mathscr{R}}_{j}$ using a fourth-order numerical quadrature.

As in MISDC methods [4], conservative MISDC methods handle the non-stiff advection process explicitly and integrate the stiff diffusion and reaction processes implicitly. (The stiffness of the diffusion term grows as $\mathrm{O}\left(\Delta t / \Delta x^{2}\right)$. In the present implementation, $\Delta t$ scales with $\Delta x$ (i.e., $\mathrm{O}(\Delta t)=\mathrm{O}(\Delta x)$ ); thus, with a sufficiently refined spatial grid, or in regions of high spatial resolution in an adaptive mesh refinement method, the diffusion term can be stiff even with a small diffusivity $\mu$. The same is true for applications that choose $\Delta t$ independently of $\Delta x$.) The time steps used for the integration of the advection and diffusion processes are larger than that of the reaction process. To advance the solution of the spatially-discretized A-D-R equations by one time step, these processes are integrated sequentially using methods that are first order in time. Then the accuracy of the solution is improved by iteratively solving a series of correction equations, which simultaneously reduce splitting and integration errors. 
In the temporal discretization, let $\Delta t>0$ be the time step and $t_{n}=n \Delta t$, for $n=0,1,2, \ldots$, be the $n$th time-level. Consider for now a simplified case in which the same time step $\Delta t$ is used for all processes. To advance the solution of (12) by one time step, the following equations are approximated sequentially:

$$
\begin{aligned}
& \bar{u}_{\mathrm{A}}(t+\Delta t)=\bar{u}(t)+\int_{t}^{t+\Delta t} \overline{\mathscr{A}}\left(\tau, \bar{u}_{\mathrm{A}}(\tau)\right) \mathrm{d} \tau \\
& \bar{u}_{\mathrm{D}}(t+\Delta t)=\bar{u}(t)+\int_{t}^{t+\Delta t}\left(\overline{\mathscr{A}}\left(\tau, \bar{u}_{\mathrm{A}}(\tau)\right)+\overline{\mathscr{D}}\left(\tau, \bar{u}_{\mathrm{D}}(\tau)\right)\right) \mathrm{d} \tau, \\
& \bar{u}(t+\Delta t)=\bar{u}(t)+\int_{t}^{t+\Delta t}\left(\overline{\mathscr{A}}\left(\tau, \bar{u}_{\mathrm{A}}(\tau)\right)+\overline{\mathscr{D}}\left(\tau, \bar{u}_{\mathrm{D}}(\tau)\right)+\overline{\mathscr{R}}(\tau, \bar{u}(\tau))\right) \mathrm{d} \tau .
\end{aligned}
$$

Note that when the same $\Delta t$ is used for all processes, $\bar{u}_{\mathrm{D}}=\bar{u}_{\mathrm{A}}+\int_{t}^{t+\Delta t} \overline{\mathscr{D}} \mathrm{d} \tau$ and $\bar{u}=\bar{u}_{\mathrm{D}}+\int_{t}^{t+\Delta t} \overline{\mathscr{R}} \mathrm{d} \tau$. However, these relationships do not hold when different time steps are used for the processes.

The above approach avoids the solution of a system that couples the non-local $\overline{\mathscr{D}}$ term and the nonlinear $\bar{R}$ term, which may be computationally expensive. Moreover, the intermediate solutions are weakly coupled in (16)-(18): the solution $\bar{u}_{\mathrm{A}}$ of (16) is used in the evaluation of $\overline{\mathscr{A}}$ in (17) and (18), and the solution $\bar{u}_{\mathrm{D}}$ of (17) is used in the evaluation of $\overline{\mathscr{D}}$ in (18). This approach generates approximations that are more accurate than the traditional operator-splitting approach $[18,28]$, in which processes are completely decoupled and integrated sequentially.

Given some interval $\left[t_{n}, t_{n+1}\right]$ on which the solution is sought and an approximation $\tilde{\bar{u}}(t)$ to $\bar{u}(t)$, conservative MISDC methods improve the accuracy of $\tilde{\tilde{u}}(t)$ by computing an approximation to the correction term

$$
\delta(t) \equiv \bar{u}(t)-\tilde{\bar{u}}(t) .
$$

To this end, let $E(t, \tilde{\bar{u}})$ be the residual function associated with $\tilde{\bar{u}}(t)$ given by

$$
E(t, \tilde{\bar{u}})=\bar{u}\left(t_{n}\right)+\int_{t_{n}}^{t}(\overline{\mathscr{A}}(\tau, \tilde{\bar{u}}(\tau))+\overline{\mathscr{D}}(\tau, \tilde{\bar{u}}(\tau))+\overline{\mathscr{R}}(\tau, \tilde{\bar{u}}(\tau))) \mathrm{d} \tau-\tilde{\bar{u}}(t) .
$$

Following [4], the correction equation that arises from (18) and (20) is

$$
\begin{aligned}
\delta(t)= & \int_{t_{n}}^{t}(\overline{\mathscr{A}}(\tau, \tilde{\bar{u}}(\tau)+\delta(\tau))-\overline{\mathscr{A}}(\tau, \tilde{\bar{u}}(\tau))+\overline{\mathscr{D}}(\tau, \tilde{\bar{u}}(\tau)+\delta(\tau))-\overline{\mathscr{D}}(\tau, \tilde{\bar{u}}(\tau)) \\
& +\overline{\mathscr{R}}(\tau, \tilde{\bar{u}}(\tau)+\delta(\tau))-\overline{\mathscr{R}}(\tau, \tilde{\bar{u}}(\tau))) \mathrm{d} \tau+E(t, \tilde{\bar{u}}) .
\end{aligned}
$$

Given a sth order approximate solution $\tilde{\bar{u}}$ (i.e., $\|\bar{u}-\tilde{\tilde{u}}\|=\mathrm{O}\left(\Delta t^{s}\right)$ ) on the time interval $\left[t_{n}, t_{n+1}\right]$, if $\overline{\mathscr{A}}, \overline{\mathscr{D}}$, and $\overline{\mathscr{R}}$ are Lipschitz continuous in $\bar{u}$, then (21) implies that $\|\delta(t)-E(t, \tilde{\bar{u}})\|=\mathrm{O}\left(\Delta t^{s+1}\right)$. Thus, from a $(s+1)$ th order approximation for $E(t, \tilde{\tilde{u}})$ and a simple first-order rectangle rule approximation to the integral on the right side of (21), an approximation $\tilde{\delta}(t)$ to $\delta(t)$ can be computed such that $\|\delta(t)-\tilde{\delta}(t)\|=\mathrm{O}\left(\Delta t^{s+1}\right)$. The approximate correction $\tilde{\delta}$ can then be used to update $\tilde{\bar{u}}$ to yield a $(s+1)$ th order approximation to $\bar{u}$. Thus, in the case where $\overline{\mathscr{A}}, \overline{\mathscr{D}}$, and $\overline{\mathscr{R}}$ are Lipschitz continuous, the error bounds can be vigorously proven.

However, in the context of the A-D-R equation, the operators $\overline{\mathscr{A}}$ and $\overline{\mathscr{D}}$ approximate differential operators, which are not Lipschitz continuous. Therefore, additional assumptions must be made about the provisional solutions $\tilde{\bar{u}}$ (or, equivalently, $\overline{\mathscr{A}}(\tilde{\bar{u}})$ and $\overline{\mathscr{D}}(\tilde{\bar{u}})$ ) for the above arguments to hold; see [1,4,5,24] for a discussion on the interaction between temporal and spatial errors. Moreover, in conservative MISDC 
methods, limiters may be applied in the computation of the left and right states and spatial derivatives. The application of limiters may also render these operators non-Lipschitz continuous and affect the accuracy and stability of the methods. A solution to the difficulty with limiters is described near the end of this section.

By solving (21), the accuracy of an approximate solution $\tilde{\bar{u}}$ is improved. However, a direct solution of (21) requires an approximation of a system of coupled nonlinear equations. Thus, following the approach used to obtain (16)-(18), a weakly coupled set of correction equations are approximated sequentially instead:

$$
\begin{aligned}
\delta_{\mathrm{A}}(t)= & \int_{t_{n}}^{t}\left(\overline{\mathscr{A}}\left(\tau, \tilde{\bar{u}}(\tau)+\delta_{\mathrm{A}}(\tau)\right)-\overline{\mathscr{A}}(\tau, \tilde{\bar{u}}(\tau))\right) \mathrm{d} \tau+E(t, \tilde{\bar{u}}), \\
\delta_{\mathrm{D}}(t)= & \int_{t_{n}}^{t}\left(\overline{\mathscr{A}}\left(\tau, \tilde{\bar{u}}(\tau)+\delta_{\mathrm{A}}(\tau)\right)-\overline{\mathscr{A}}(\tau, \tilde{\bar{u}}(\tau))+\overline{\mathscr{D}}\left(\tau, \tilde{\bar{u}}(\tau)+\delta_{\mathrm{D}}(\tau)\right)-\overline{\mathscr{D}}(\tau, \tilde{\bar{u}}(\tau))\right) \mathrm{d} \tau+E(t, \tilde{\bar{u}}), \\
\delta(t)= & \int_{t_{n}}^{t}\left(\overline{\mathscr{A}}\left(\tau, \tilde{\bar{u}}(\tau)+\delta_{\mathrm{A}}(\tau)\right)-\overline{\mathscr{A}}(\tau, \tilde{\bar{u}}(\tau))+\overline{\mathscr{D}}\left(\tau, \tilde{\bar{u}}(\tau)+\delta_{\mathrm{D}}(\tau)\right)-\overline{\mathscr{D}}(\tau, \tilde{\bar{u}}(\tau))\right. \\
& +\overline{\mathscr{R}}(\tau, \tilde{\bar{u}}(\tau)+\delta(\tau))-\overline{\mathscr{R}}(\tau, \tilde{\bar{u}}(\tau))) \mathrm{d} \tau+E(t, \tilde{\bar{u}}) .
\end{aligned}
$$

In addition to splitting errors, solutions computed by conservative MISDC methods contain integration errors that arise from numerical quadrature approximation of the integrals. The integration errors associated with the stiff reaction term may be larger than the advection and diffusion errors. Now owing to the decoupling of processes, it is possible to selectively reduce integration errors by using smaller time steps for fast-scale processes. Thus, the time step used to integrate the reaction process is smaller than those of advection and diffusion processes. For gas dynamics applications in which the advection and diffusion time scales are similar, the advection and diffusion time steps are taken to be equal.

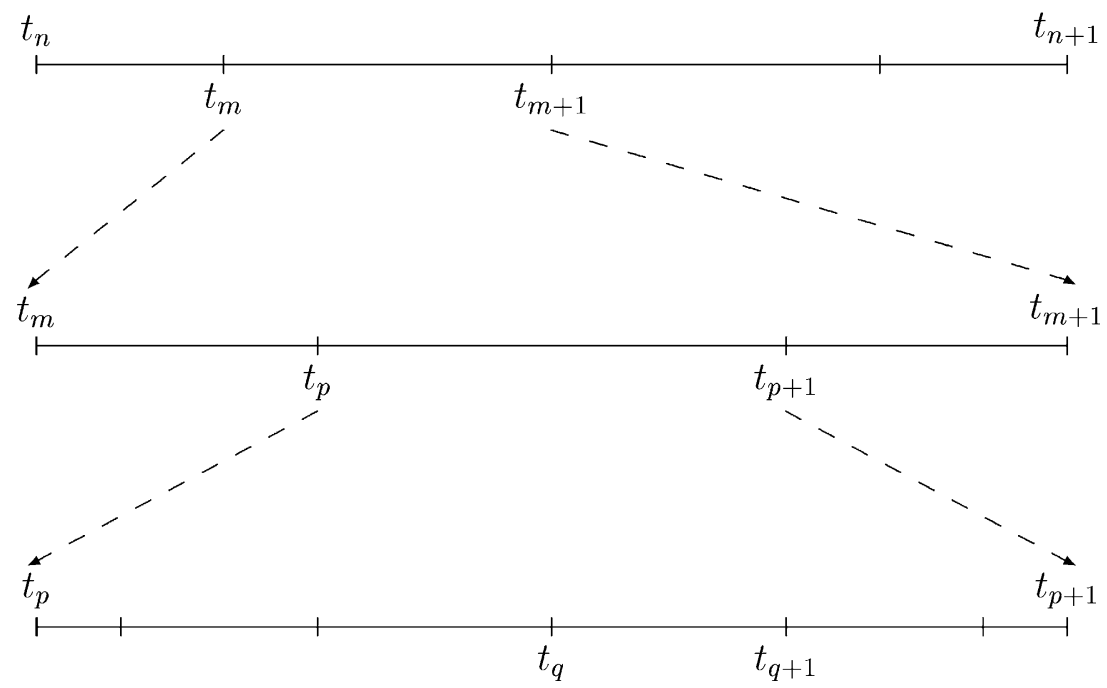

Fig. 1. Illustration of three levels of time-step subdivision: $\left[t_{n}, t_{n+1}\right]$ into $\left[t_{m}, t_{m+1}\right],\left[t_{m}, t_{m+1}\right]$ into $\left[t_{p}, t_{p+1}\right]$, and $\left[t_{p}, t_{p+1}\right]$ into $\left[t_{q}, t_{q+1}\right]$. In this example, $N_{\mathrm{A}}=4, N_{\mathrm{D}}=3, N_{\mathrm{R}}=6, m=1, p=1$, and $q=3$. 
To integrate the solution from $t_{n}$ to $t_{n+1}$, the time interval $\left[t_{n}, t_{n+1}\right]$ is divided into subintervals. Fig. 1 shows an example of time-step subdivision. Below is a description of the time-step subdivision for the general case in which reaction is stiffer than diffusion, which is in turn stiffer than advection. The time interval $\left[t_{n}, t_{n+1}\right]$ is divided into $N_{\mathrm{A}}$ subintervals by choosing points $t_{n, m}$ for $m=0,1, \ldots, N_{\mathrm{A}}$ such that $t_{n}=t_{n, 0}<t_{n, 1}<\cdots<t_{n, m}<\cdots<t_{n, N_{\mathrm{A}}}=t_{n+1}$. To use smaller time steps for the diffusion process and yet smaller ones for the reaction process, the subinterval $\left[t_{n, m}, t_{n, m+1}\right]$ is subdivided into $N_{\mathrm{D}}$ subintervals by choosing points $t_{n, m, p}$ for $p=0,1, \ldots, N_{\mathrm{D}}$ such that $t_{n, m}=t_{n, m, 0}<t_{n, m, 1}<\cdots<t_{n, m, p}<\cdots<t_{n, m, N_{\mathrm{D}}}=t_{n, m+1}$. $\left(N_{\mathrm{D}}=1\right.$ corresponds to the case where the advection and diffusion time steps are equal.) Then $\left[t_{n, m, p}, t_{n, m, p+1}\right]$ is further subdivided into $N_{\mathrm{R}}$ subintervals by choosing points $t_{n, m, p, q}$ for $q=0,1, \ldots, N_{\mathrm{R}}$ such that $t_{n, m, p}=t_{n, m, p, 0}<t_{n, m, p, 1}<\cdots<t_{n, m, p, q}<\cdots<t_{n, m, p, N_{\mathrm{R}}}=t_{n, m, p+1}$. For notational simplicity, the subscript $n$ in $t_{n, m}$ is omitted, $n$ and $m$ omitted in $t_{n, m, p}$, and $n, m$, and $p$ omitted in $t_{n, m, p, q}$ where there is no ambiguity, i.e., $t_{n, m}, t_{n, m, p}$, and $t_{n, m, p, q}$ are written as $t_{m}, t_{p}$, and $t_{q}$, respectively. Let $\Delta t_{m} \equiv t_{m+1}-t_{m}, \Delta t_{p} \equiv t_{p+1}-t_{p}$, and $\Delta t_{q} \equiv t_{q+1}-t_{q}$. In this implementation, $t_{m}, t_{p}$, and $t_{q}$ are Gauss-Lobatto nodes of the intervals $\left[t_{n}, t_{n+1}\right]$, $\left[t_{m}, t_{m+1}\right]$, and $\left[t_{p}, t_{p+1}\right]$, respectively.

For an arbitrary function $\psi(t)$, let $\psi_{m}^{k}, \psi_{p}^{k}$, and $\psi_{q}^{k}$ denote the numerical approximations to $\psi\left(t_{m}\right), \psi\left(t_{p}\right)$, and $\psi\left(t_{q}\right)$, respectively, after $k$ deferred correction iterations. Using this notation, a multi-implicit discretization of the correction equations (22)-(24), based on forward and backward Euler methods, is given by

$$
\begin{aligned}
\delta_{\mathrm{A} m+1}^{k}= & \delta_{\mathrm{A} m}^{k}+\Delta t_{m}\left(\overline{\mathscr{A}}_{m}\left(\bar{u}_{m}^{k}+\delta_{\mathrm{A} m}^{k}\right)-\overline{\mathscr{A}}_{m}\left(\bar{u}_{m}^{k}\right)\right)+E_{m+1}\left(\bar{u}^{k}\right)-E_{m}\left(\bar{u}^{k}\right) \\
\delta_{\mathrm{D}_{p+1}}^{k}= & \delta_{\mathrm{D}_{p}}^{k}+\Delta t_{p}\left(\overline{\mathscr{A}}_{m}\left(\bar{u}_{m}^{k}+\delta_{\mathrm{A} m}^{k}\right)-\overline{\mathscr{A}}_{m}\left(\bar{u}_{m}^{k}\right)+\overline{\mathscr{D}}_{p+1}\left(\bar{u}_{p+1}^{k}+\delta_{\mathrm{D}_{p+1}}^{k}\right)-\overline{\mathscr{D}}_{p+1}\left(\bar{u}_{p+1}^{k}\right)\right)+E_{p+1}\left(\bar{u}^{k}\right)-E_{p}\left(\bar{u}^{k}\right) \\
\delta_{q+1}^{k}= & \delta_{q}^{k}+\Delta t_{q}\left(\overline{\mathscr{A}}_{m}\left(\bar{u}_{m}^{k}+\delta_{\mathrm{A} m}^{k}\right)-\overline{\mathscr{A}}_{m}\left(\bar{u}_{m}^{k}\right)+\overline{\mathscr{D}}_{p+1}\left(\bar{u}_{p+1}^{k}+\delta_{\mathrm{D} p+1}^{k}\right)-\overline{\mathscr{D}}_{p+1}\left(\bar{u}_{p+1}^{k}\right)\right. \\
& \left.+\overline{\mathscr{R}}_{q+1}\left(\bar{u}_{q+1}^{k}+\delta_{q+1}^{k}\right)-\overline{\mathscr{R}}_{q+1}\left(\bar{u}_{q+1}^{k}\right)\right)+E_{q+1}\left(\bar{u}^{k}\right)-E_{q}\left(\bar{u}^{k}\right) .
\end{aligned}
$$

As in [4,22], the discretized correction equations (25)-(27) are rewritten in terms of updated values of the target function. To this end, let $I_{s}^{s+1}\left(\psi^{k}\right)$ denote the numerical quadrature approximation to

$$
\int_{t_{s}}^{t_{s+1}}\left(\overline{\mathscr{A}}\left(\tau, \bar{\psi}^{k}(\tau)\right)+\overline{\mathscr{D}}\left(\tau, \bar{\psi}^{k}(\tau)\right)+\overline{\mathscr{R}}\left(\tau, \bar{\psi}^{k}(\tau)\right)\right) \mathrm{d} \tau,
$$

where $s=m, p$, or $q$, and $\psi$ denotes an arbitrary function. Since the quadrature must be done for each interval $\left[t_{s}, t_{s+1}\right]$, there are $N_{s}=N_{\mathrm{A}}, N_{\mathrm{D}}$, and $N_{\mathrm{R}}$ quadrature rules, for $s=m, p$, and $q$, respectively. The quadrature is given by

$$
I_{s}^{s+1}\left(\psi^{k}\right)=\sum_{l=0}^{N_{S}} \alpha_{s}^{l}\left(\overline{\mathscr{A}}\left(t_{l}, \bar{\psi}^{k}\left(t_{l}\right)\right)+\overline{\mathscr{D}}\left(t_{l}, \bar{\psi}^{k}\left(t_{l}\right)\right)+\overline{\mathscr{R}}\left(t_{l}, \bar{\psi}^{k}\left(t_{l}\right)\right)\right)
$$

for $s=0, \ldots, N_{s}$. The coefficients $\alpha_{s}^{l}$ are precomputed, and the quadrature is reduced to matrix-vector multiplications.

The correction terms $\delta_{\mathrm{A}}, \delta_{\mathrm{D}}$, and $\delta$ are used to obtain $\bar{u}_{\mathrm{A}}, \bar{u}_{\mathrm{D}}$, and $\bar{u}$, respectively, from $\tilde{\bar{u}}$. That is, $\bar{u}_{\mathrm{A}}$ is set to $\tilde{\bar{u}}+\delta_{\mathrm{A}}, \bar{u}_{\mathrm{D}}$ to $\tilde{\bar{u}}+\delta_{\mathrm{D}}$, and $\bar{u}$ to $\tilde{\bar{u}}+\delta$. Combining these update relations with (26) and (27), one arrives at the following update equations for the diffusion and reaction processes: 


$$
\begin{aligned}
\bar{u}_{D_{p+1}}^{k+1}= & \bar{u}_{D_{p}}^{k+1}+\Delta t_{p}\left(\overline{\mathscr{A}}_{m}\left(\bar{u}_{A_{m}}^{k+1}\right)-\overline{\mathscr{A}}_{m}\left(\bar{u}_{m}^{k}\right)+\overline{\mathscr{D}}_{p+1}\left(\bar{u}_{D_{p+1}}^{k+1}\right)-\overline{\mathscr{D}}_{p+1}\left(\bar{u}_{p+1}^{k}\right)\right)+I_{p}^{p+1}\left(\bar{u}^{k}\right) \\
\bar{u}_{q+1}^{k+1}= & \bar{u}_{q}^{k+1}+\Delta t_{q}\left(\overline{\mathscr{A}}_{m}\left(\bar{u}_{A_{m}}^{k+1}\right)-\overline{\mathscr{A}}_{m}\left(\bar{u}_{m}^{k}\right)+\overline{\mathscr{D}}_{p+1}\left(\bar{u}_{D_{p+1}}^{k+1}\right)-\overline{\mathscr{D}}_{p+1}\left(\bar{u}_{p+1}^{k}\right)+\overline{\mathscr{R}}_{q+1}\left(\bar{u}_{q+1}^{k+1}\right)-\overline{\mathscr{R}}_{q+1}\left(\bar{u}_{q+1}^{k}\right)\right) \\
& +I_{q}^{q+1}\left(\bar{u}^{k}\right) .
\end{aligned}
$$

Because the advection process is treated explicitly, $\bar{u}_{\mathrm{A}}^{k}$ need not be computed; thus, an update equation for $\bar{u}_{\mathrm{A}}^{k}$ is not needed.

In summary, the following steps improve the order of accuracy of $\bar{u}^{k}$ by one:

For $m=0, \ldots, N_{\mathrm{A}}-1$

For $p=0, \ldots, N_{\mathrm{D}}-1$

Solve (30) for $\bar{u}_{D_{p+1}+1}^{k+1}$ Compute $\overline{\mathscr{D}}_{p+1}\left(\bar{u}_{D_{p+1}}^{k+1}\right)$

For $q=0, \ldots, N_{\mathrm{R}}-1$

End

Solve (31) for $\bar{u}_{q+1}^{k+1}$; Compute $\overline{\mathscr{R}}_{q+1}\left(\bar{u}_{q+1}^{k+1}\right)$

Update $\overline{\mathscr{D}}_{p+1}\left(\bar{u}_{p+1}^{k+1}\right)$

End

Compute $\overline{\mathscr{A}}_{m+1}\left(\bar{u}_{m+1}^{k+1}\right)$

\section{End}

Forward and backward Euler methods are used to compute the provisional solution $\bar{u}^{0}$. That is, when $k=-1$, the terms $\overline{\mathscr{A}}_{m}\left(\bar{u}_{m}^{-1}\right), \overline{\mathscr{D}}_{p+1}\left(\bar{u}_{p+1}^{-1}\right), \overline{\mathscr{R}}_{q+1}\left(\bar{u}_{q+1}^{-1}\right)$, and the numerical quadratures $I$ 's in (30) and (31) are taken to be zero. Alternatively, a more accurate provisional solution may be computed by means of a higher-order integration method or using numerical quadratures from previous time steps.

Because $\overline{\mathscr{A}}, \overline{\mathscr{D}}$, and $\overline{\mathscr{R}}$ are discrete approximations to non-Lipschitz operators, to insure convergence special care must be taken when applying limiters in the computation of the left and right states and the spatial derivatives. As previously noted, given a $(s+1)$ th order approximation $\tilde{\bar{u}}$, the terms inside the integral of the right side of (21) are $\mathrm{O}\left(\Delta t^{s+1}\right)$. However, if limiters are applied to $\bar{A}^{k}$ at a spatial location $x_{j}$ but not to $\overline{\mathscr{A}}^{k+1}$ (or vice versa), then the difference $\left\|\mathscr{A}^{k+1}-\overline{\mathscr{A}}^{k}\right\|$ may be large at $x_{j}$; similar problems may arise for $\overline{\mathscr{D}}$ and $\overline{\mathscr{R}}$. To overcome this difficulty, in the computation of the provisional solution $(k=-1)$, the spatial locations at which limiters are applied are recorded. Then during the SDC iterations $(k \geqslant 0)$, the limiters are only applied at those locations. The numerical examples in Section 4 show that, with this approach, the conservative MISDC methods preserve sharp gradients in solutions. Nonetheless, it is conceivable that the first-order temporal errors in the provisional solutions may lead to incorrect limiter locations. Alternative approaches of determining limiter locations are discussed in Section 5. However, it must be acknowledged that none of these approaches have been rigorously shown to yield correct limiter locations.

\subsection{Reacting gas dynamics}

The remainder of this section extends the conservative MISDC methods to the system of reacting flow (1)-(4).

In the diffusion step, the advection and diffusion processes are coupled, with the advection processes handled explicitly and the diffusion processes integrated implicitly. First, the explicit (advective) components are computed. Thus, to compute the provisional solution $(k=-1)$ the following equations are first solved:

$$
\begin{aligned}
& \rho_{t}+(\rho u)_{x} \equiv \rho_{t}-\mathscr{A}(\rho)=0, \\
& (\rho u)_{t}+\left(\rho u^{2}+p\right)_{x} \equiv(\rho u)_{t}-\mathscr{A}(\rho u)=0,
\end{aligned}
$$




$$
\begin{aligned}
& (\rho E)_{t}+(\rho u E+u p)_{x} \equiv(\rho E)_{t}-\mathscr{A}(\rho E)=0, \\
& \left(\rho Z_{i}\right)_{t}+\left(\rho u Z_{i}\right)_{x} \equiv\left(\rho Z_{i}\right)_{t}-\mathscr{A}\left(\rho Z_{i}\right)=0 .
\end{aligned}
$$

For notational simplicity, in the definition of the advective flux terms, the parameters $\rho, \rho u, \rho E$, and $\rho Z_{i}$ are also used as indices for the flux functions, e.g., $\mathscr{A}(\rho) \equiv \mathscr{A}_{\rho}(\rho) \equiv-(\rho u)_{x}$; similar notation is also used for $\mathscr{D}$ and $\mathscr{R}$ below. The $k$ th SDC iteration is given by

$$
\begin{aligned}
& \left(\bar{\rho}_{\mathrm{A}}\right)_{p+1}^{k+1}=(\bar{\rho})_{p}^{k+1}+\Delta t_{p}\left(\overline{\mathscr{A}}(\bar{\rho})_{m}^{k+1}-\overline{\mathscr{A}}(\bar{\rho})_{m}^{k}\right)+I_{p}^{p+1}\left(\rho^{k}\right), \\
& (\overline{\rho u})_{p+1}^{k+1}=(\overline{\rho u})_{p}^{k+1}+\Delta t_{p}\left(\overline{\mathscr{A}}(\overline{\rho u})_{m}^{k+1}-\overline{\mathscr{A}}(\overline{\rho u})_{m}^{k}\right)+I_{p}^{p+1}\left(\rho u^{k}\right), \\
& \left(\bar{\rho} E_{\mathrm{A}}\right)_{p+1}^{k+1}=(\overline{\rho E})_{p}^{k+1}+\Delta t_{p}\left(\overline{\mathscr{A}}(\overline{\rho E})_{m}^{k+1}-\overline{\mathscr{A}}(\overline{\rho E})_{m}^{k}\right)+I_{p}^{p+1}\left(\rho E^{k}\right), \\
& \left(\bar{\rho} Z_{i \mathrm{~A}}\right)_{p+1}^{k+1}=\left(\bar{\rho} Z_{i}\right)_{p}^{k+1}+\Delta t_{p}\left(\overline{\mathscr{A}}\left(\bar{\rho} Z_{i}\right)_{m}^{k+1}-\overline{\mathscr{A}}\left(\bar{\rho} Z_{i}\right)_{m}^{k}\right)+I_{p}^{p+1}\left(\rho Z_{i}^{k}\right) .
\end{aligned}
$$

The diffusion processes are then integrated using the intermediate solutions of (36)-(39) as initial values. Following the approach in [7], the following equations are solved:

$$
\begin{aligned}
& \rho_{t}=0 \equiv \mathscr{D}(\rho), \\
& (\rho u)_{t}=\left(\mu u_{x}\right)_{x} \equiv \mathscr{D}(\rho u), \\
& \left(\rho \frac{u^{2}}{2}\right)_{t}=\left(\mu\left(\frac{u^{2}}{2}\right)_{x}\right)_{x} \equiv \mathscr{D}\left(\rho \frac{u^{2}}{2}\right), \\
& (\rho T)_{t}=\frac{R}{\gamma-1}\left(\lambda T_{x}\right)_{x} \equiv \mathscr{D}(\rho T), \\
& \left(\rho Z_{i}\right)_{t}=\left(D Z_{i} x\right)_{x} \equiv \mathscr{D}\left(\rho Z_{i}\right) .
\end{aligned}
$$

The update equations for the diffusion processes are:

$$
\begin{aligned}
& \left(\bar{\rho}_{\mathrm{D}}\right)_{p+1}^{k+1}=\left(\bar{\rho}_{\mathrm{A}}\right)_{p+1}^{k+1}+\Delta t_{p}\left(\overline{\mathscr{D}}(\bar{\rho})_{p+1}^{k+1}-\overline{\mathscr{D}}(\bar{\rho})_{p+1}^{k}\right), \\
& \left(\bar{\rho} \overline{\mathrm{D}}_{\mathrm{D}}\right)_{p+1}^{k+1}=(\overline{\rho u})_{p+1}^{k+1}+\Delta t_{p}\left(\overline{\mathscr{D}}(\overline{\rho u})_{p+1}^{k+1}-\overline{\mathscr{D}}(\overline{\rho u})_{p+1}^{k}\right), \\
& \left(\bar{\rho} \frac{\bar{u}^{2}}{2}\right)_{p+1}^{k+1}=\left(\rho \frac{\bar{u}^{2}}{2}\right)_{p+1}^{k+1}+\Delta t_{p}\left(\overline{\mathscr{D}}\left(\rho \frac{u^{2}}{2}\right)_{p+1}^{k+1}-\overline{\mathscr{D}}\left(\rho \frac{\bar{u}^{2}}{2}\right)_{p+1}^{k}\right), \\
& \left.(\overline{\rho T})_{\mathrm{D}}\right)_{p+1}^{k+1}=\left(\bar{\rho} T_{\mathrm{A}}\right)_{p+1}^{k+1}+\Delta t_{p}\left(\overline{\mathscr{D}}(\overline{\rho T})_{p+1}^{k+1}-\overline{\mathscr{D}}(\overline{\rho T})_{p+1}^{k}\right), \\
& \left(\bar{\rho} \bar{Z}_{i \mathrm{D}}\right)_{p+1}^{k+1}=\left(\bar{\rho} Z_{i \mathrm{~A}}\right)_{p+1}^{k+1}+\Delta t_{p}\left(\overline{\mathscr{D}}\left(\bar{\rho} Z_{i}\right)_{p+1}^{k+1}-\overline{\mathscr{D}}\left(\bar{\rho} Z_{i}\right)_{p+1}^{k}\right) .
\end{aligned}
$$




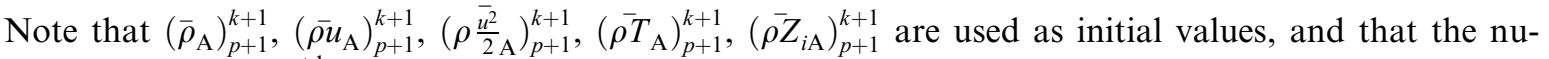
merical quadratures $I_{p}^{p+1}$ 's, which are included in (36)-(39) do not appear in (45)-(49). Further details for the solution of (45)-(49) are described in Appendix A.

Because there is no reaction associated with the density, momentum, or the kinetic or pressure component of the total energy, the solutions of (45)-(48) are taken to be the solutions for the $k$ th SDC iteration; i.e., $\left.(\bar{\rho})_{p+1}^{k+1}=\left(\bar{\rho}_{\mathrm{D}}\right)_{p+1}^{k+1},(\overline{\rho u})_{p+1}^{k+1}=(\overline{\rho u})_{\mathrm{D}}\right)_{p+1}^{k+1},\left(\rho \frac{u^{2}}{2}\right)_{p+1}^{k+1}=\left(\rho \frac{u^{2}}{2}\right)_{p+1}^{k+1}$, and $(\overline{\rho T})_{p+1}^{k+1}=\left(\overline{\rho T} T_{\mathrm{D}}\right)_{p+1}^{k+1}$. The mass fraction $\left(\rho \bar{Z}_{i}\right)_{p+1}^{k+1}$ and total energy $(\rho E)_{p+1}^{k+1}$ are updated after the reaction step.

In the reaction step, the equation

$$
\begin{aligned}
\left(\rho Z_{i}\right)_{q+1}^{k+1}= & \left(\rho Z_{i}\right)_{q}^{k+1}+\Delta t_{q}\left(\overline{\mathscr{A}}\left(\rho Z_{i}\right)_{m}^{k+1}-\overline{\mathscr{A}}\left(\rho Z_{i}\right)_{m}^{k}+\overline{\mathscr{D}}\left(\rho Z_{i \mathrm{D}}\right)_{p+1}^{k+1}-\overline{\mathscr{D}}\left(\rho Z_{i \mathrm{D}}\right)_{p+1}^{k}\right. \\
& \left.+\mathscr{R}\left(\rho Z_{i}\right)_{q+1}^{k+1}-\mathscr{R}\left(\rho Z_{i}\right)_{q+1}^{k}\right)+I_{q}^{q+1}\left(\rho Z_{i}^{k}\right),
\end{aligned}
$$

is solved not at cell averages but rather at cell edges for $i=1, \ldots, M$, so that high-order spatial accuracy may be achieved. Cell-edge values for $\overline{\mathscr{A}}$ and $\overline{\mathscr{D}}$ are computed using a piecewise parabolic interpolation of the associated cell-averages as described in Section 3.1 and in [8]. The solution $\left(\rho Z_{i}\right)_{q+1}^{k+1}$ is then used to compute cell-averaged values for $\left(\bar{\rho} \bar{Z}_{i}\right)_{q+1}^{k+1}$ and to update $(\overline{\rho E})_{q+1}^{k+1}$.

Compared to the operator-splitting approach, which is a typical approach for computing solutions to models of reacting gas dynamics, the conservative MISDC methods offer the same benefits of allowing the stiff reaction terms to be solved implicitly while avoiding the solution of coupled nonlinear equations, and of allowing different time steps to be used for different processes. Furthermore, unlike traditional operatorsplitting approaches, for which temporal accuracy higher than second order is difficult to achieve, conservative MISDC methods can be constructed to yield high-order accuracy. To compute high-order solutions, additional computational cost is required by the iterative process. In Section 4.4, the efficiency of the third- and fourth-order conservative MISDC methods is compared to a conservative, second-order operator-splitting method.

\section{Numerical examples}

In this section, the stability, accuracy, and convergence behavior of the conservative MISDC methods are studied using test cases with increasing complexity. The Euler equations are used to test the ability of the methods to robustly handle shocks; the reactive, compressible Euler equations are used to study the accuracy of the methods in the solution of a Chapman-Jouguet (CJ) detonation wave; and the reactive, compressible Navier-Stokes equations are used to study the accuracy of the methods in obtaining solutions in which the diffusion and reaction terms are balanced to generate continuous solutions with sharp gradients. The last example is also used to compare the efficiency of the methods to a conservative, secondorder operator-splitting method. In all calculations reported in this section, $R=1$. Solid-wall boundary conditions are assumed on both ends of the spatial domain. All computations reported in this section were performed using Fortran programs implemented in double precision on an IBM system with an Intel Pentium IV $2 \mathrm{GHz}$ processor and with $1 \mathrm{~GB}$ of RAM.

\subsection{Euler equations}

In the first example, a well-studied problem consisting of the Euler equations of gas dynamics for a polytropic gas [21] is solved to study the representation of shocks by the conservative MISDC methods and the restriction on time step imposed by the advective component of the equations. The initial conditions are 
$(\rho, u, p)= \begin{cases}\left(1,0,10^{3}\right), & x<0.1 \\ \left(1,0,10^{-2}\right), & 0.1 \leqslant x<0.9, \\ \left(1,0,10^{2}\right), & 0.9 \leqslant x \leqslant 1 .\end{cases}$

An inviscid and non-reactive model is considered, i.e., the diffusion constants $\mu, \lambda$, and $D$, and the heat release $q_{i}$ are taken to be 0 . Because the flow is non-reactive, the mass fraction equation (4) is not solved. Eqs. (1)-(3) are solved on the spatial domain $[0,1]$ and time domain $[0,0.038]$.

Figs. 2 and 3 display results at $t=0.038$ computed using the third-order conservative MISDC method with $N_{\mathrm{A}}=2$. Numerical solutions computed using 200 and 400 cells (shown with crosses) are compared to one that is computed using 800 cells (shown with solid lines), which is regarded as a reference solution. These resolutions were chosen to facilitate direct comparison with results reported in [21,26]. The time step is taken to be $\Delta t=2 \Delta t_{m}=0.067 \Delta x$, which corresponds to a Courant-Friedrichs-Lewy (CFL) number, given by $\mathrm{CFL}=\max (|u \pm c|) \Delta t_{m} / \Delta x$, of $\sim 1$. Some smearing is observed with 200 cells, but in the solution obtained with 400 cells most of the fine structures are captured, even though the methods are not designed for non-stiff and non-split problems such as the Euler equations. Indeed, these solutions compare favorably to results reported in [21].

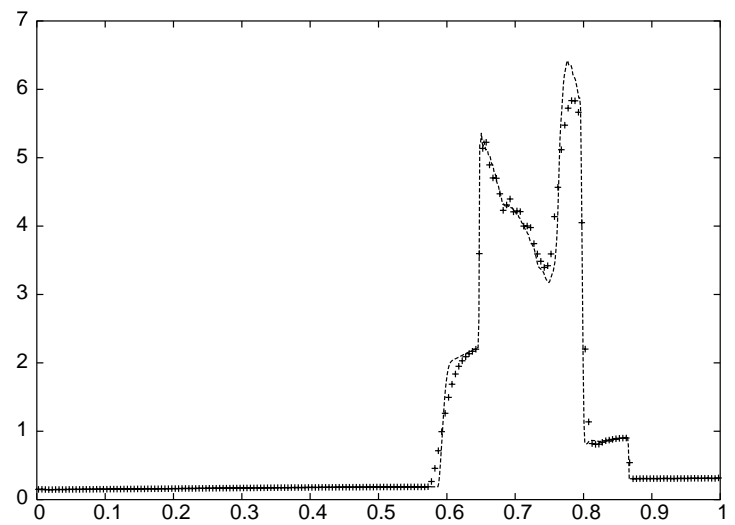

(a)

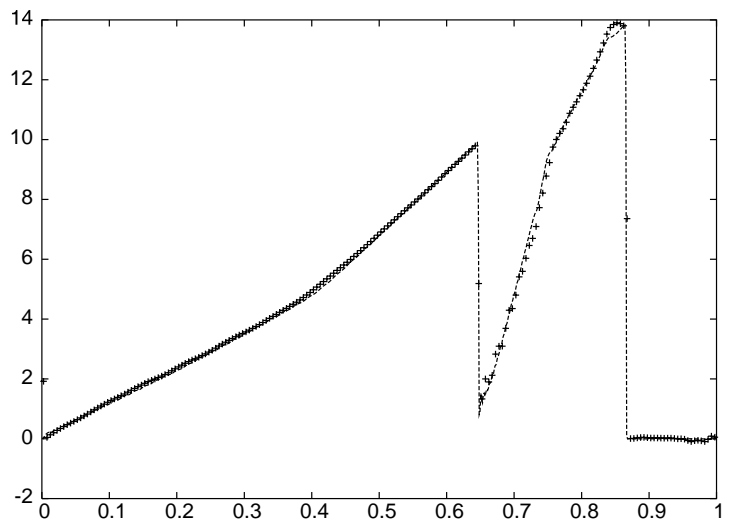

(b)

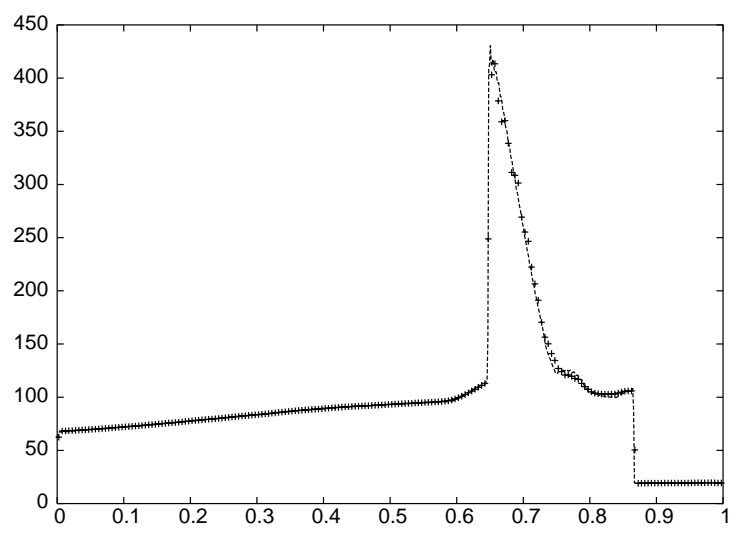

(c)

Fig. 2. Numerical results for example 1 using 200 cells: (a) density; (b) velocity; (c) pressure. Crosses and dashed lines are solutions computed using 200 and 800 cells, respectively. 

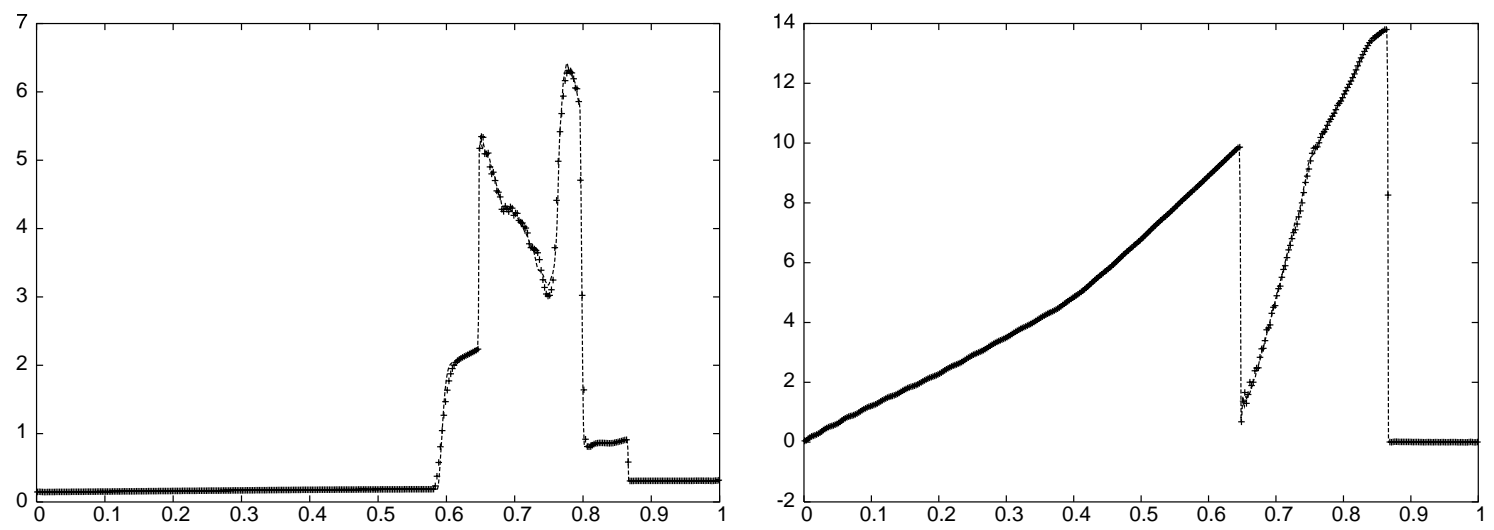

(a)

(b)

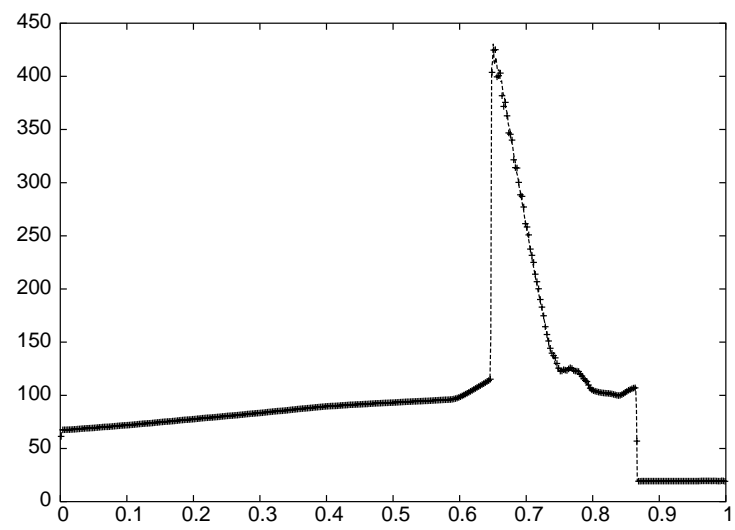

(c)

Fig. 3. Numerical results for example 1 using 400 cells: (a) density; (b) velocity; (c) pressure. Crosses and dashed lines are solutions computed using 400 and 800 cells, respectively.

\subsection{Compressible Euler equations for a reacting mixture}

The next example assesses the accuracy of the methods in computing a solution to a CJ detonation wave. The example considers a standard simplified form of the reacting mixture, in which a species may be of two states, unburnt and burnt. The unburnt gas is converted to burnt gas by a one-step irreversible chemical reaction; thus, $M=1$ and the subscript $i$ for the chemical species is omitted. The Arrhenius kinetics in (4) is replaced by the Heaviside kinetics

$$
g(T, Z)= \begin{cases}B Z, & T \geqslant T_{\mathrm{o}} \\ 0, & T<T_{\mathrm{o}}\end{cases}
$$

Because diffusion is assumed negligible in this system, the diffusion coefficients in model Eqs. (1)-(4) are set to 0 . The initial conditions consist of totally burnt gas $(Z=0)$ on the left-hand side $(-0.5 \leqslant x<0)$ and totally unburnt gas $(Z=1)$ on the right-hand side $(0 \leqslant x \leqslant 0.5)$. The density, velocity, and pressure of the burnt gas are given by $\rho=1.4, u=0$, and $p=1$; and the corresponding initial values of the unburnt gas are given by $\rho=0.887565, u=-0.577350$, and $p=0.191709$. These states are connected by a CJ detonation wave moving with speed 1 . The reaction rate is $B=10^{2}$, ignition temperature $T_{\mathrm{o}}=0.22$, and heat release 
constant $q_{0}=1$. If the reaction zone is not resolved by a sufficiently refined spatial grid, the present methods may not generate a correct approximation of the detonation wave. In particular, with the parameters specified above, because the ignition temperature is only slightly higher than the temperature of the unburnt gas $(T=0.2160)$, if the reaction zone is not sufficiently resolved, the temperature may not be calculated with sufficiently high accuracy, which may result in incorrect burning rate and wave propagation speed.

Eqs. (1)-(4) are integrated by means of a third-order conservative MISDC method with $N_{\mathrm{A}}=2$ and $N_{\mathrm{R}}=1$. The reaction process is integrated analytically as done in $[7,13,19]$. A spatial grid of 256 cells is used. Fig. 4 shows the pressure $p$ of the detonation wave and the normalized fuel concentration $Z$ at $t=2$. These results show that the conservative MISDC methods correctly capture the propagation of this detonation wave.

The parameters and results for the above simulation are similar to those reported in [13]. The reaction could be made much stiffer by choosing higher reaction rates such as $B \geqslant 10^{3}$. Then unless spatial resolution is refined accordingly, the method produces a non-physical solution, a discontinuity that propagates with a speed of one mesh cell per time step. This phenomenon was first reported by Colella et al. [7]. In order to avoid this problem, one could use adaptive mesh refinement or specialized front tracking schemes (e.g., $[14,19])$. These approaches will be pursued in the context of conservative MISDC methods in future studies.

\subsection{Compressible Navier-Stokes equations for a multi-species reacting mixture}

The third example considers a reacting model with three species $(M=3)$ in a viscous medium. This is the only example considered in this study that involves multiple species undergoing advection, diffusion, and reaction. This example is used (i) to show the accuracy of the methods in capturing sharp gradients using a coarse grid, (ii) to demonstrate temporal convergence of the methods, and (iii) to show that the high-order methods are more efficient than a second-order operator-splitting method.

The parameters for this system are $T_{\mathrm{o}}=2, B=10^{6}, \alpha=0, q_{1}=100, q_{2}=0, q_{3}=0, w_{1}=2, w_{2}=32$, $w_{3}=18, v_{r 1}=2, v_{r 2}=1, v_{r 3}=0, v_{p 1}=0, v_{p 2}=0, v_{p 3}=2$, and $\mu=\lambda=D=2 \times 10^{-4}$. The initial data is

$$
\psi=\psi_{1}+\psi_{2}\left(1-\tanh \left(\frac{x}{0.005}\right)\right)
$$

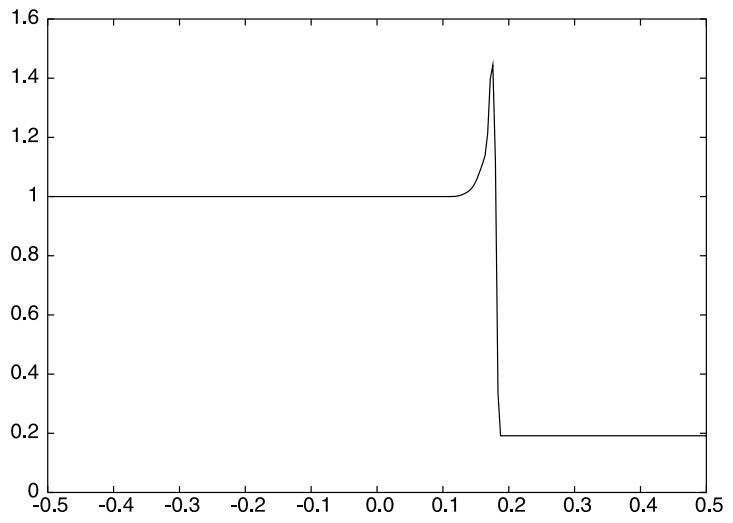

(a)

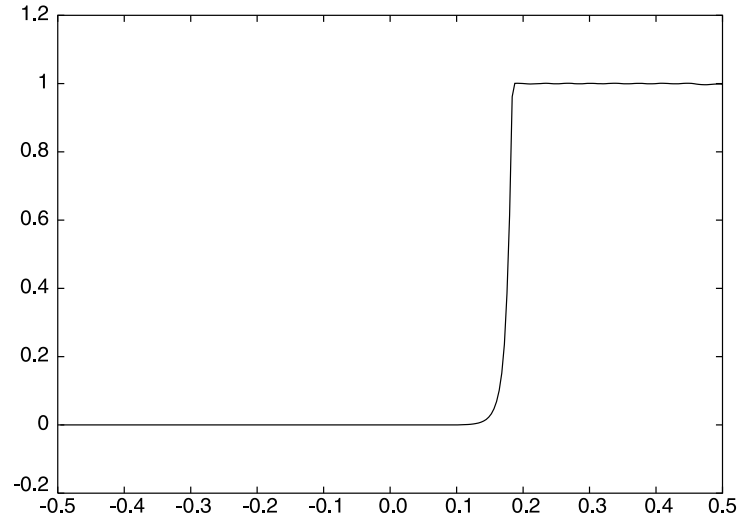

(b)

Fig. 4. Numerical results for example 2: (a) pressure; (b) normalized fuel concentration. 
where $\psi=\rho, u, p, Z_{1}, Z_{2} ;\left(\rho_{1}, \rho_{2}\right)=(1,0.5),\left(u_{1}, u_{2}\right)=(0,4),\left(p_{1}, p_{2}\right)=(1,9.5),\left(Z_{11}, Z_{12}\right)=(0,1 / 18)$, and $\left(Z_{21}, Z_{22}\right)=(0,4 / 9) ; Z_{3}=1-Z_{1}-Z_{2}$. These parameters were chosen to facilitate a direct comparison with one of the test cases in [3].

Eqs. (1)-(4) are integrated by means of a third-order conservative MISDC method with $N_{\mathrm{A}}=2, N_{\mathrm{D}}=1$, and $N_{\mathrm{R}}=20$. Fig. 5 shows the solutions at $t=0.05$. A numerical solution computed using 100 cells (displayed with crosses) is compared to the reference solution computed using 800 cells (displayed with solid lines). Even with a coarse spatial grid of 100 cells, the solutions have sharp gradients. The time step is taken to be $\Delta t=0.1 \Delta x$, which corresponds to a CFL number of $\sim 1$. These results show that even at low spatial resolution, the conservative MISDC method correctly captures the propagation of the waves.

The next set of tests focus on the convergence behavior of the conservative MISDC methods using the same set of parameters as described above. A spatial grid of 800 cells is used. With this more refined spatial grid, the fronts are resolved. Because limiters affect the accuracy of a solution, they are not applied. Eqs. (1) -(4) are integrated by means of conservative MISDC methods with $N_{\mathrm{A}}+1=K, N_{\mathrm{D}}=1$, and $N_{\mathrm{R}}=20$, for $K=3,4$, and 5, i.e., methods with orders 3, 4, and 5. Fig. 6 shows $L_{\infty}$ errors of the density $(\rho)$ at $t=0.05$ using time step $\Delta t=\Delta x / 2^{r+3}$, for $r=0,1,2,3$. Because no analytic solution is known for the system, the numerical solution computed using the fifth-order conservative MISDC method with $r=4$ is used as the

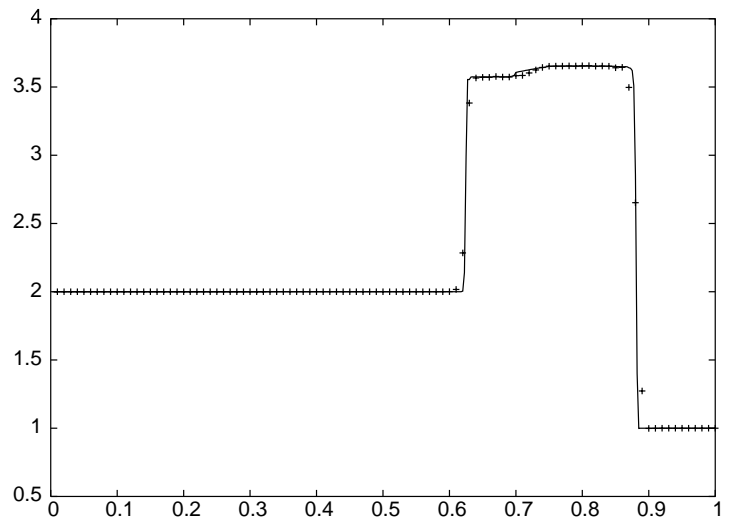

(a)

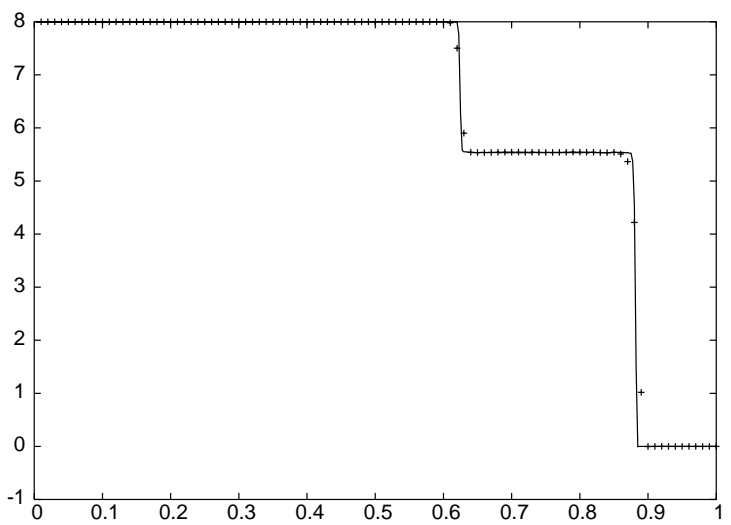

(c)

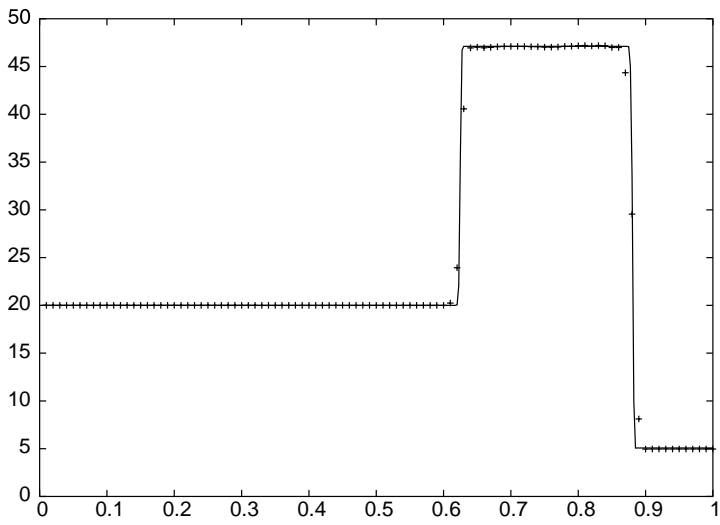

(b)

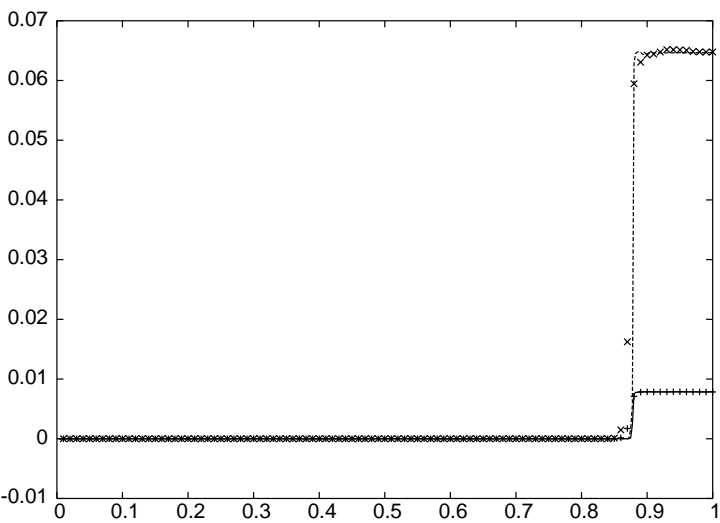

(d)

Fig. 5. Numerical results for example 3: (a) density; (b) pressure; (c) velocity; (d) reactant mass fractions. Crosses and solid lines are solutions computed using 100 and 400 cells, respectively. 


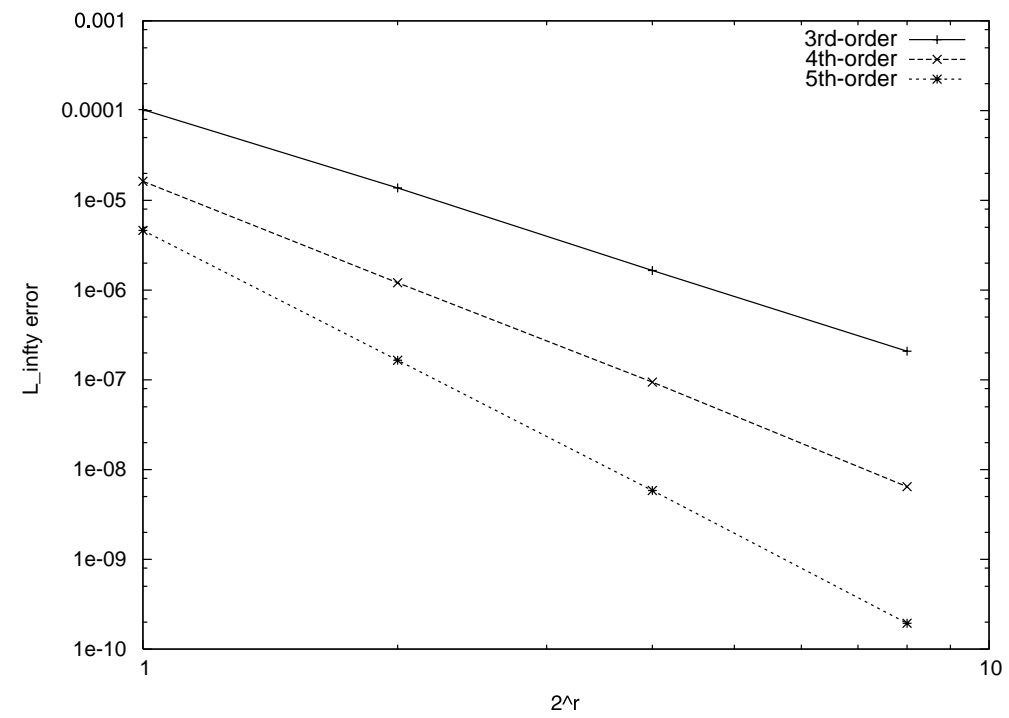

Fig. 6. Log- $\log$ plot of $L_{\infty}$ error versus $2^{r}$, where $\Delta t=2^{r} \Delta x$ for conservative MISDC methods with orders 3,4 , and 5.

reference solution in computing the errors. In all cases, the approximations converge at the expected rate $K$ : magnitudes of the least-square slopes for the curves in Fig. 6 are found to be 2.9884, 3.7598, and 4.8451, for $K=3$, 4, and 5, respectively. Similar convergence results were also obtained for other variables.

\subsection{Efficiency comparison with a second-order operator-splitting method}

The previous example is also used to compare the efficiency of the third- and fourth-order conservative MISDC methods to a conservative, second-order operator-splitting method (hereafter "second-order OS method"), which may be considered a typical approach for solving gas dynamics problems. The secondorder OS method is similar to the fractional step method described in [7], except that a fourth-order method is used to compute conservative cell-edge fluxes (thus, the method, like the conservative MISDC methods, is fourth-order in space) and that a smaller time step is used for the reaction process. (The same time step is used for all processes in [7].) Let $L_{\mathrm{A}}^{\Delta t}, L_{\mathrm{D}}^{\Delta t}$, and $L_{\mathrm{R}}^{\Delta t}$ be the integration operators that advance the advection, diffusion, and reaction processes, respectively, by $\Delta t$. Then, using the Strang splitting approach the secondorder OS method advances the solution $U$ from $t_{n}$ to $t_{n+1}$ by

$$
U^{n+1}=L_{\mathrm{A}}^{\frac{\Delta t}{2}} L_{\mathrm{D}}^{\frac{\Delta t}{2}} L_{\mathrm{R}}^{\frac{\Delta t}{20}} \cdots L_{\mathrm{R}}^{\frac{\Delta t}{20}} L_{\mathrm{D}}^{\frac{\Delta t}{2}} L_{\mathrm{A}}^{\frac{\Delta t}{2}} U^{n} .
$$

The reaction integration operator $L_{\mathrm{R}}^{\frac{\Delta t}{20}}$ is applied 20 times.

Fig. 7 shows a $\log -\log$ plot of $L_{\infty}$ error of $\rho$ versus computation times, measured in seconds, for the second-order OS method, and for the third- and fourth-order conservative MISDC methods. These results were obtained using a spatial grid of 800 cells and time step $\Delta t=\Delta x / 2^{r+3}$, for $r=0,1,2,3$. The errors were measured at $t=0.05$ using the fifth-order conservative MISDC method with $r=4$ as the reference solution. These results indicate that, for all temporal resolutions considered, the third- and fourth-order conservative MISDC methods are more efficient than the second-order OS method, and that, for this problem, the fourth-order method is more efficient than the third-order method if an accuracy of $\leqslant 10^{-6}$ is desired.

However, compared to the second-order OS method, the conservative MISDC methods may have higher memory cost. At each fractional time step, the second-order OS method requires function values at the 


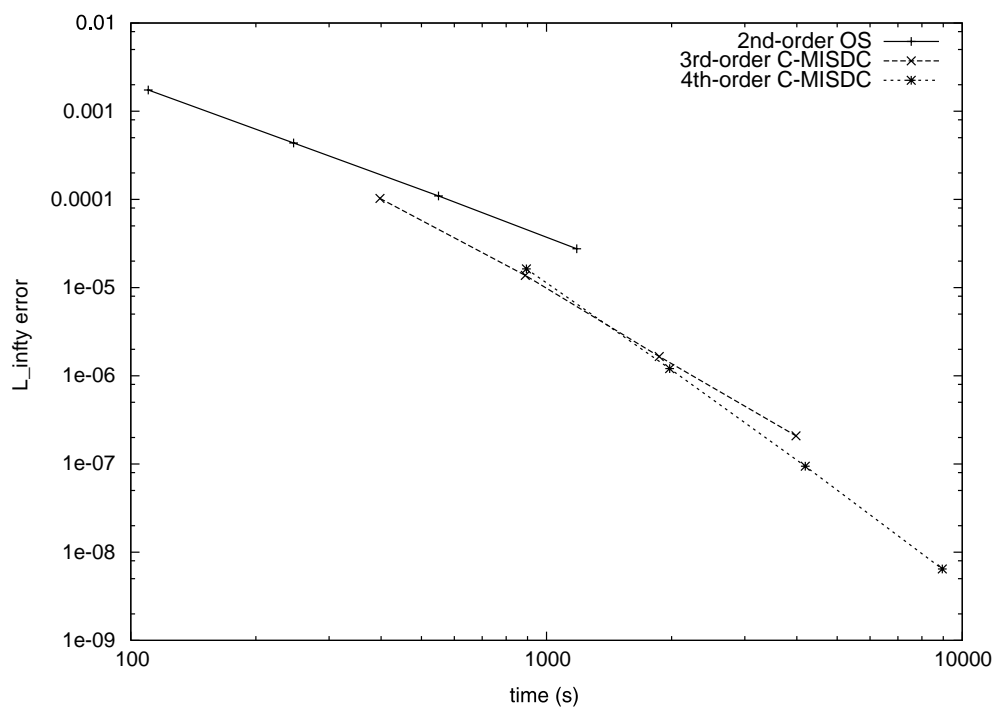

Fig. 7. $\log -\log$ plot of $L_{\infty}$ error versus computation times, measured in seconds, for a second-order OS method, and the third- and fourth-order conservative MISDC method.

previous fractional time step; thus, the method has a memory cost of $\mathrm{O}(N)$, where $N$ is the number of spatial subintervals. The correction steps of a conservative MISDC method require function values at each substep of the previous iteration, which gives a memory cost of $\mathrm{O}\left(N \times\left(N_{\mathrm{A}}\left(1+N_{\mathrm{D}}\left(1+N_{\mathrm{R}}\right)\right)\right)\right)$. Which method is more desirable, given their memory costs and computational efficiency, depends on the application and on available resources.

\section{Discussion}

In this paper, conservative MISDC methods for the temporal integration of reacting gas dynamics, which involve processes with multiple time scales, have been presented. When applied to such models, conservative MISDC methods integrate the advection term explicitly, and the diffusion and reaction terms implicitly, independently, and possibly with different time steps. Unlike the original MISDC methods [4], the advection and diffusion processes are integrated in flux difference form to insure correct propagation of shocks or sharp gradients. Unlike standard operator-splitting methods, MISDC methods can easily be constructed to generate numerical solutions with arbitrarily high-order of temporal accuracy. This is achieved by simultaneously reducing integration and splitting errors in the deferred correction iterations.

When the third-order conservative MISDC method is applied to the Euler equations in Section 4.1, the maximum advection time step $\Delta t_{m}$ allowed by stability is the same as the maximum time step allowed by the Godunov method [11] or PPM [8]. Because three SDC iterations are required for the third-order conservative MISDC method, the ratio of $\Delta t_{m}$ to the number of Riemann solves is $\Delta t_{m} / 3$. (In general, the ratio is $\Delta t_{m} / K$ for a $K$ th-order MISDC method.) This ratio can be used as an efficiency measure. Compared to a ratio of 1 for the Godunov method or PPM, one may conclude that the conservative MISDC methods offer little advantage in this problem. Indeed, the methods offer the most possible benefit for applications with stiff reactions (e.g., the reactive Navier-Stokes equations in Section 4.3) and these methods are not intended for a non-stiff and non-split problem such as the Euler equations. Nevertheless, this example illustrates that the methods are as effective as many popular methods in capturing shock propagations. 
The target applications for the conservative MISDC methods are those in which the reaction operator is much stiffer than the advection and diffusion operators and in which solving the decoupled diffusion and reaction problems is much simpler than solving the coupled diffusion-reaction equation. In particular, the reactions in some applications are computed using chemistry packages, the black-box nature of which renders solving coupled diffusion-reaction equations difficult. With a decoupling of the diffusion and reaction terms, the conservative MISDC methods provide a solution for incorporating such black-box solvers into a highly accurate time integration scheme.

In the examples considered in this study, diffusion is assumed to be either negligible or small. Thus, the advection and diffusion time steps are chosen to be equal. However, conservative MISDC methods can also be applied to problems with diffusion that is stiffer than advection (e.g., low Mach number reacting flows [17]). When applied to these applications, the diffusion time step should be smaller than the advection time step. Such problems have been studied in [4] for the original MISDC methods.

In the current implementation, limiter locations are computed during the computation of the provisional solution; then, to ensure convergence, limiters are applied only at those locations during the SDC iterations. Because a first-order method is used in the computation of the provisional solutions, the resulting limiter locations may be inaccurate. Alternatively, one may recompute the limiter locations at the final SDC iteration, which should yield a more accurate solution, and repeat the time step using those locations. However, because the solution at that final SDC iteration was obtained using limiter locations that (possibly) contain large errors, the limiter locations recomputed from this solution may also contain large errors. Another alternative is to iterate the above process (i.e., the time step is repeated until the limiter locations recomputed from the final SDC correction step is the same as those in the previous iteration of the time step); however, convergence (or convergence to correct limiter locations) of such iterations is not guaranteed. While the current implementation has been shown to preserve sharp gradients in solutions, the development of a more rigorous approach to determining limiter locations is certainly a worthwhile challenge.

\section{Appendix A}

Two issues involved in the solution of (45)-(49) deserve special attention. First, The kinetic component of the total energy, $\left(\rho \frac{\bar{u}^{2}}{2} \mathrm{~A}\right)_{p+1}^{k+1}$, which is needed in (47), is not given explicitly as an intermediate solution by (36)-(39). Second, the target conserved quantities in (46)-(49) are $\bar{\rho} u_{\mathrm{D}}, \rho \frac{u^{2}}{2} \mathrm{D}, \bar{\rho} \bar{T}_{\mathrm{D}}$, and $\bar{\rho} \bar{Z}_{i \mathrm{D}}$, respectively, whereas the diffusive fluxes are functions of $u_{\mathrm{D}}, T_{\mathrm{D}}$, and $Z_{i \mathrm{D}}$ only and are independent of $\rho$. Thus, standard solvers for equations of the form $f-q f_{x x}=g$ cannot be applied to (46)-(49).

A solution to the first problem is as follows. The kinetic energy $\left(\rho \frac{\bar{u}^{2}}{2} \mathrm{~A}\right)_{p+1}^{k+1}$ is computed from the density $\left(\bar{\rho}_{\mathrm{A}}\right)_{p+1}^{k+1}$ and momentum $\left(\overline{\rho u_{\mathrm{A}}}\right)_{p+1}^{k+1}$, which are given by (36) and (37), respectively. The temperature term $\left(\rho T_{\mathrm{A}}\right)_{p+1}^{k+1}$, which appears on the right side of (48), is computed from $\left(\rho E_{\mathrm{A}}\right)_{p+1}^{k+1},\left(\rho \frac{u^{2}}{2 \mathrm{~A}}\right)_{p+1}^{k+1}$, and $\left(\bar{\rho} Z_{i \mathrm{~A}}\right)_{p+1}^{k+1}$ using (6).

A solution to the second problem is now described. First, note that because the density Eq. (1) has neither diffusion nor reaction term, $(\bar{\rho})_{p+1}^{k+1}=\left(\bar{\rho}_{\mathrm{D}}\right)_{p+1}^{k+1}$; thus, (45) is superfluous and $\left(\bar{\rho}_{\mathrm{D}}\right)_{p+1}^{k+1}$ is a known quantity. The procedures for approximating the solution of (46) are as follows. As noted previously, because the diffusion term is not a scalar multiple of the second-derivative of the target quantity $\bar{\rho} u_{\mathrm{D}}$, standard solvers cannot be used. Thus, to approximate a solution to (46), one solves

$$
\left(\overline{\rho u}_{\mathrm{D}}\right)_{p+1}^{k+1}-\frac{\Delta t_{p}}{\Delta x} \mu\left(L_{\mathrm{D}}^{+}\left(\left(\bar{\rho} u_{\mathrm{D}}\right)_{p+1}^{k+1},\left(\bar{\rho}_{\mathrm{D}}\right)_{p+1}^{k+1}\right)-L_{\mathrm{D}}^{-}\left(\left(\overline{\rho u_{\mathrm{D}}}\right)_{p+1}^{k+1},\left(\bar{\rho}_{\mathrm{D}}\right)_{p+1}^{k+1}\right)\right)=\left(\overline{\rho u_{\mathrm{A}}}\right)_{p+1}^{k+1}
$$

where $L_{\mathrm{D}}^{+}$and $L_{\mathrm{D}}^{-}$are linear operators that approximate $\left(u_{\mathrm{D}}\right)_{x}^{k+1} p+1$ at $\left\{x_{j}\right\}_{j=1}^{N}$ and $\left\{x_{j}\right\}_{j=0}^{N-1}$, respectively, using $\left(\bar{\rho} u_{\mathrm{D}}\right)_{p+1}^{k+1}$ and $(\bar{\rho})_{p+1}^{k+1}$. This is done by first computing cell-edge values of $\rho u_{\mathrm{D}}$ and $\rho$, dividing to obtain 
$u_{\mathrm{D}_{p+1}^{k+1}}^{k}$ at cell edges, then approximating $\left(u_{\mathrm{D}}\right)_{x_{p+1}}^{k+1}$ at cell edges using centered difference. The solution of (47)(49) is similar to that of (46).

\section{References}

[1] S. Abarbanel, D. Gottlieb, M.H. Carpenter, On the removal of boundary errors caused by Runge-Kutta integration of nonlinear partial differential equations, SIAM J. Sci. Comput. 17 (1996) 777-782.

[2] U.M. Ascher, L.R. Petzold, Computer Methods for Ordinary Differential Equations and Differential-Algebraic Equations, SIAM, Philadelphia, PA, 2000.

[3] W. Bao, S. Jin, The random projection method for stiff multispecies detonation capturing, J. Comput. Phys. 178 (2002) $37-57$.

[4] A. Bourlioux, A.T. Layton, M.L. Minion, High-order multi-implicit spectral deferred correction methods for problems of reactive flow, J. Comput. Phys. 189 (2) (2003) 651-675.

[5] M.H. Carpenter, D. Gottlieb, S. Abarbanel, W.-S. Don, The theoretical accuracy of Runge-Kutta time discretization for the initial boundary value problem: a study of the boundary error, SIAM J. Sci. Comput. 16 (1995) 1241-1252.

[6] P. Colella, Multidimensional upwind methods for hyperbolic conservation laws, J. Comput. Phys. 87 (1990) 171-200.

[7] P. Colella, A. Majda, V. Roytburd, Theoretical and numerical structure for reacting shock waves, SIAM J. Sci. Stat. Comput. 7 (4) (1986) 1059-1080.

[8] P. Colella, P.R. Woodward, The piecewise parabolic method (PPM) for gas-dynamical simulations, J. Comput. Phys. 54 (1984) 174-201.

[9] C.F. Curtiss, J.O. Hirschfelder, Integration of stiff equations, Proc. Natl. Acad. Sci. 38 (1952) 235.

[10] A. Dutt, L. Greengard, V. Rokhlin, Spectral deferred correction methods for ordinary differential equations, BIT 40 (2) (2000) 241-266.

[11] S.K. Godunov, A difference method for numerical calculation of discontinuous solutions of the equations of hydrodynamics, Mat. Sbornik 47 (1959) 271-306.

[12] E. Hairer, G. Wanner, Solving Ordinary Differential Equations II, Stiff and Differential-Algebraic Problems, Springer, Berlin, 1996.

[13] F.K. Hebeker, R. Rannacher, An adaptive finite element method for unsteady convection-dominated flows with stiff source terms, SIAM J. Sci. Comput. 21 (3) (1999) 799-818.

[14] C. Helzel, R.J. LeVeque, G. Warnecke, A modified fractional step method for the accurate approximation of detonation waves, SIAM J. Sci. Comput. 22 (4) (2000) 1489-1510.

[15] R.J. Kee, F.M. Rupley, E. Meeks, J.A. Miller, CHEMKIN-III: A FORTRAN chemical kinetics for the analysis of gas-phase chemical and plasma kinetics, Sandia National Laboratories, Technical report SAND96-8216, 1996.

[16] C.A. Kennedy, M.H. Carpenter, Additive Runge-Kutta schemes for convection-diffusion-reaction equations, Appl. Numer. Math. 44 (1-2) (2002) 139-181.

[17] R. Klein, Semi-implicit extension of a Godunov-type scheme based on low Mach number asymptotics I: one-dimensional flow, J. Comput. Phys. 121 (1995) 213-237.

[18] D. Lanser, J.G. Verwer, Analysis of operator splitting for advection-diffusion-reaction problems from air pollution modelling, J. Comput. Appl. Math. 111 (1-2) (1999) 201-216.

[19] R.J. LeVeque, K.-M. Shyue, One-dimensional front tracking based on high resolution wave propagation methods, SIAM J. Sci. Comput. 16 (2) (1995) 348-377.

[20] R.J. LeVeque, K.-M. Shyue, Two-dimensional front tracking based on high resolution wave propagation methods, J. Comput. Phys. 123 (1996) 354-368.

[21] R. Liska, B. Wendroff, Comparison of several difference schemes on 1D and 2D test problems for the Euler equations, Technical Report LA-UR-01-6225, LANL, Los Alamos, 2001.

[22] M.L. Minion, Semi-implicit spectral deferred correction methods for ordinary differential equations, Commun. Math. Sci. 1 (3) (2003) 471-500.

[23] S. Osher, S. Chakravarthy, High resolution schemes and the entropy condition, SIAM J. Numer. Anal. 21 (1984) $984-995$.

[24] R.D. Richtmyer, K.W. Morton, Difference methods for initial value problems, second ed., Wiley Interscience, New York, 1967.

[25] C.-W. Shu, S. Osher, Efficient implementation of essentially non-oscillatory shock-capturing schemes, J. Comput. Phys. 77 (1988) 439-469.

[26] C.-W. Shu, S. Osher, Efficient implementation of essentially non-oscillatory shock-capturing schemes, II, J. Comput. Phys. 83 (1989) 32-78.

[27] G. Strang, On the construction and comparison of difference schemes, SIAM J. Numer. Anal. 8 (3) (1968) $506-517$.

[28] N.N. Yanenko, The Method of Fractional Steps, Springer, New York, 1971. 